\title{
Discussion
}

\section{Artificial intelligence: Immediate promises and ultimate goals}

Leonard UHR

University of Wisconsin, Computer Science Department, Madison, WI 53706, USA

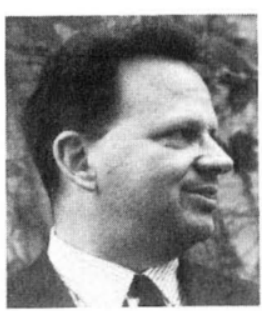

Leonard Uhr was born in Philadelphia (PA) in 1927. He received the B.A. degree from Princeton University in 1949 and the Ph.D. from the University of Michigan in 1957. From 1957 to 1964 he was an Assistant Research Scientist and Research Scientist at the Mental Health Research Institute and a Lecturer and Associate Professor in the Psychology Department at the University of Michigan, Ann Arbor. From 1964 to the present he has been a Professor in the Computer Sciences

Department at the University of Wisconsin, Madison.

His chief research interests center around the long-term goal of developing precise, testable theoretical models of perceptual and cognitive processes, focussing on the following problems: visual perception of ordinary real-world scenes of objects; perception (in real time) of objects in motion; learning by inductive inference and hypothesis-formation; modelling of simple systems that cycle through the whole perceptual-cognitive-motor loop; and the design and programming of large parallel-serial arrays and networks structured into an 'anatomy' appropriate to the algorithms they must execute.

He has written the books Pattern Recognition, Learning and Thought (Prentice-Hall, 1973) and Algorithm-Structured Computer Arrays and Networks (Academic Press, 1984) and edited the books Pattern Recognition (Wiley, 1966) and Multi-Computer Arrays and Networks for Image Processing (with K. Preston, Jr., Academic Press, 1982) and a number of papers.

Today's computers come with 'software' programs that are typically so difficult to use and to learn that 'artificial intelligence' and 'human engineering' can play important roles in making computers more accessible. Computers are rapidly becoming so large and powerful, and so cheap, that the large AI programs needed are becoming increasingly feasible. But the ultimate promise of artificial intelligence is far greater - to throw light on and perhaps solve some of the very difficult key problems of intelligence. It would be a tragic irony if today's fad-of-the-computer and 'AI expert systems' for 'smarts' left even fewer people trying to work on these fundamental problems.

North-Holland

Human Systems Management 5 (1985) 155-158
Keywords: Artificial intelligence, real intelligence, VLSI, multi-computer networks, 'user friendly systems', 'smarts', 'human engineering', fads and fallacies, modelling intelligent cognition.

\section{The great ultimate promise of artificial intelligence}

Artificial intelligence is, ultimately, the development of a theoretical understanding of (intelligent) thinking. Once developed, this understanding can then be applied to a myriad of wonderful projects, from making computers 'smarter', more 'user friendly' and even 'sympathetic', to building robots that cook, clean up, keep accounts, win at the races and beat the stock market, build cars for us and drive them, listen to our troubles, tell us jokes, solve our financial, intellectual and personal problems, and in general lavish us with all the free time and friendship we can handle and help us to enjoy it and to use it productively.

Today's computers are unfriendly, dumb and awkward to use

Despite all the glossy, sexy visual displays and inspirational music, we are not all that close to such goals. What we do have is rapidly increasing numbers of cheaper and cheaper $\$ 500, \$ 2000$, $\$ 5000$ or $\$ 8000$ 'personal computers' - the Commodores, Macintoshes, IBM-PCs, and Lisas - that most sane people find difficult, awkward, and/or limited, if not frightening, to punch away at.

Computers have not been designed or programmed to be used by ordinary people, people who have little or not desire to program them or spend their lives trying to understand obscure and often incorrect directions in poorly written and disorganized manuals. So a small but growing number of programmers are trying to make them more 'user friendly' and 'smart'. What this typi- 
cally means is that the computer is programmed to display a few bad pictures of paintbrushes and wastebaskets, or a few idiot directions when the user types some magic symbols (e.g., 'help' or '?' or ' $\mathrm{H}$ ' or god-knows-what - 'see page 237' of some unreadable System Manual Appendix for further obfuscation).

In such situations a little bit of artificial intelligence could go a long way toward making the dialog more tolerable.

Computers are increasingly powerful, hence increasingly idle

Every 14 to 26 months the number of basic components (transistors, logic gates) that can be packed onto a single VLSI (Very Large Scale Integration) chip doubles in size, and this means that computers, which are built from chips, double in power. Today's personal computer-workstations, as we all have been told but find hard to believe, are therefore more powerful than the multi-million dollar supercomputers of the 1950s and 1960 s - even at the same time that, in comparison with the state of the art, they are quite old-fashioned and slow, if not obsolete.

Computers 5 or 10 times faster still could be built and marketed today (if mass-produced in large quantities) for less money, as Apple's $\$ 2500$ Macintosh, and (probably by the time this is published) Commodore's \$1200? and Sinclair's \$500 new computers are beginning to demonstrate. In 5, 10 , and 20 years, computers 5, 40 and 1000 times more powerful still will cost even less.

\section{Artificial intelligence can fill many idle moments with 'smarts'}

What on earth will we do with such computers? Not everybody wants something whirring in deep silence at our desks searching for the next prime number. There would probably be nothing nicer than to have computers be intelligent. If our personal computer is many thousands of times more powerful than it needs to be to sit there waiting for us to type in the next few words or numbers, why not have it work during all that idle time at being intelligent?

Computers are so awkward and difficult to use that we can improve their behavior strikingly (or give the illusion of striking improvements) with additions that are little more than simple common sense and simple good will. We supposedly make 'dumb' terminals 'smart' by giving them a 'mouse' (a toy car we roll around on our desk to move the cursor-pointer on the computer's display screen). We supposedly can make can't-do-anything brute hardware 'learn' by giving it software programs that will 'tell' it how to do anything conceivable. (We know theoretically that this is possible, if we only knew how to write the necessary learning programs; but in truth today we know very little about how to get computers to learn.)

That the new computers are intelligent is not true at all today, except in the glossy ads. If the apocalyptic Macintosh ads and puffery reviews in the advertising-stuffed-and-supported glossy popular computer monthlies don't say it now, they shortly will - this new computer is 'brilliant' in the ways it teaches everyone how to use itself, overlays the windows, pulls down the window shades, lays on the icons and, in general, offers a rousing good time. Some of this is straightforward systems programming, some is improved human factors and industrial engineering, much is programmers with good will and friendly dispositions. A little bit is artificial intelligence, but at its very simplest and most primitive.

Fortunately for those free-lancing with high technology, most manuals and instructions for today's computers are so poorly written and disorganized that a whole new industry of books explaining how to use each new computer, and each new software package, is almost guaranteed to spring up. Why not simply improve upon the manuals, instructions, operating systems, languages, and software tools, instead of trying to make progress toward solving very difficult artificial intelligence problems? That of course is easier said than done; but that is basically what is happening today. We can call this 'artificial intelligence' and earn the field some credit for practicality.

More powerful computers can be used for limited intelligence

In 10,20 , and 30 years the very smallest, cheapest several-hundred-dollar computer will be at least 100,1000 , and then 10000 times more powerful than today's personal computers. But today's personal computers already sit idle most of the time, 
waiting for someone to type something in. Today this idle time is being filled with 'smarts' and graphics, with windows, icons, and pseudo-English words and phrases.

Today's computers sit idle only $95 \%$, occasionally $80 \%$ or $50 \%$ and sometimes even a bit smaller percent of the time. Soon they will sit idle $99 \%$ and 99.99\% of the time. That is, each personal computer-workstation in the home will be able to do thousands of times more work than is asked of it. What better use of all this potential power than to make the computer a little more friendly, smart, and intelligent?

The development has scarcely begun of systems that are truely user-friendly, flexible, responsive, and easy and pleasant to use while carrying out truely productive tasks where the human being benefits fully from the computer's potential powers. It is rather like the early days of the automobile, when drivers had to be their own mechanics. Most of us don't want to learn to start our cars with a crank, or even with a choke, much less change the oil, adjust the carburetor or replace the valves. But computers and programs are not only far more complex than automobiles, they are complex in strange and hidden ways.

A programmed computer can be likened to an enormous set of invisible buttons to push, with these buttons constantly changing their meanings far faster than we can push them. It is as though we had all the displays, buttons and switches in all the NASA and Military Command Centers quivering unobserved under the keyboard, with little more than disorganized, unreadable manuals to tell us how to reach the buttons, much less what to do with them.

Artificial intelligence and human engineering are probably the best hope for these systems. User-friendly programmers, human engineers, and artificial intelligensia can all help to make the many user-unfriendly systems more accessible. Much more difficult, but still possible with the larger computers available in 5 or 10 years, are systems that may recognize simple, clearly (if not stiltedly) articulated short sentences, and even simple objects input via television cameras (but almost certainly unmoving objects). But such systems should not be called intelligent, any more than a car with an automatic choke or a bell, light or voice that tells when doors are open or seatbelts are off.

\section{Multi-computers for real intelligence, both artificial} and natural

The truely exciting promise is that computers can be $100,1000,10000,100000$, and 1000000 times more powerful still! This will not be the kind of computer that we use today - a 'serial' computer with one single processor. That would be absolutely, theoretically impossible, simply because no computer can operate faster than the speed of light, and today's computers are rapidly approaching that absolute limit.

Instead, this will be accomplished by building multi-computer networks, where each multi-computer combines hundreds, thousands or millions of individual computers, and all of these computers can be programmed to work together on the same problem. These kinds of multi-computers can be made increasingly large, with no ultimate theoretical limit to their size. Nor will they be that much more expensive.

A traditional computer will end up (with mass production and mature technologies) costing $\$ 1$ to $\$ 20$ more (for the 5 to 200 VLSI chips in its processor and high speed memory) than the cost of its cabinetry (roughly $\$ 50$ ), input devices $(\$ 100)$, mass memories (\$100), plus output display and other devices (\$100). Each additional computer will add only roughly $\$ 1$ to this cost - and probably substantially less, since many computers will be put on each single chip. Instead of buying a traditional one-processor computer for $\$ 370$, we will have the option of buying a 100-processor computer for less than $\$ 470$, or a 10000 -processor computer substantially discounted from its list price of $\$ 9999$.

But what need is there for such a gigantic multi-computer network when a single personal computer-workstation is already far too powerful for its individual user's needs?

Very simply - real, true intelligence, whether artificial or natural, is overwhelming more complex, powerful and costly than the 'smarts' with which today's purportedly 'user-friendly' software make computers a bit less nontolerable. To develop truely intelligent systems we still need computers far larger than the multi-million dollar 'super-computers' of today, which are themselves thousands of times larger than the $\$ 500$ to $\$ 10000$ personal computer-workstations. Therefore when the $\$ 500$ workstation becomes as powerful as these 
super-computers it still will not suffice. But at some break-even point (I would conjecture somewhere between 1000 and 1000000 ) if we put enough of these individual computers together into a multi-computer network the raw computer hardware will be sufficient.

At that point it will be possible to have truely intelligent personal computer-workstations, and even autonomous robots. Whether these are actually achieved will depend upon whether artificial intelligence researchers have developed the necessary theoretical understanding, and formulated and programmed the necessary software. These are extremely complex and difficult problems, problems on which artificial intelligence researchers have scarcely begun to work.
I'd suggest that the real truth is that AI is an extremely difficult problem. It's simply the basic problem of psychology and philosophy - what is mind; how does it come to know about the world; how does it figure out and decide what to do? We have made progress; but whether it is little or much (or relatively little or much with respect to our elusive goals) is too early to say.

In the short run artificial intelligence can use band aides to patch up, smooth out and cover over. But these very limited short-term goals should not be allowed to interfere with progress toward the really exciting long-term prospects, of computers and robots not with artificial but with real intelligence. 